\title{
High current ionic diode using homogeneously charged asymmetric nanochannel network
}

membrane

Eunpyo Choi, Cong Wang, Gyu Tae Chang, and Jungyul Park ${ }^{*}$

Department of Mechanical Engineering, Sogang University, 35 Baekbeom-ro (Sinsu-dong),

Mapo-gu, Seoul 121-742, Korea 


\section{S1. In situ Formation of Nanochannel Networks}

\section{S1.1 Fabrication process of figure S1(a) and (b)}

Figure S1(a) shows PDMS device with shallow and deep channels by using the fabrication process as follows: Figure S2(a) to (e) shows schematic diagrams that describe the procedures for fabricating microfluidic devices with multilayered design. We fabricated microfluidic chips using the standard soft lithography: (a) The first layer for shallow channel was patterned with photolithography using the positive photoresist (AZ7220, AZ Electronic Materials Ltd.) and then the patterned shallow channel was etched by deep reactive ion etching (DRIE). The target depth of DRIE was $5 \mu \mathrm{m}$. (b) After removing the photoresist, the second layer for deep channel was patterned with photolithography using negative photoresist (SU-8, Microchem, Newton, MA) on the first layer. The target height was $100 \mu \mathrm{m}$. (c) A Polydimethylsiloxane (PDMS) precursor (Sylgard 184 Silicone Elastomer, Dow Corning) and a curing agent were mixed at a ratio of 10 to 1 , based on weight. Before the PDMS mixture was poured onto the fabricated master, the master was silanized with (tridecafluoro1,1,2,2,-tetrahydrooctyl)-1-trichlorosilane (Sigma Chemical Co., St. Louis, MO, USA) to allow easier removal of the PDMS after curing. The PDMS mixture was poured onto the master and cured at $95^{\circ} \mathrm{C}$ for $1 \mathrm{~h}$. (d) Then, the cured PDMS channel was peeled off from the master, cut and punched to connect microtubes. (e) The PDMS devices were directly bonded to a glass substrate without any surface treatment and then they were treated with oxygen plasma under $50 \mathrm{sccm}$ of $\mathrm{O}_{2}$ and $70 \mathrm{~W}$ for $40 \mathrm{~s}$ (Cute-MP, Femto Science, Korea).

Figure S1(b): The diluted nanospheres were centrifuged and re-suspended in $70 \%$ ethanol (v/v) and then $2 \mu \mathrm{L}$ of this solution was introduced into the deep channel-B by capillary pressure. 


\section{S1.2 Fabrication process of figure S1(c)}

The diluted nanoparticles at the intersection between shallow and deep channel-B experience a sudden pressure drop $\left(\Delta P_{12}\right)$, which tries to drag the solution in the deep channel into the shallow channel $\left(F_{\text {shallow }}\right) .{ }^{1}$ When this flow of solution in shallow channel is located at the neck (interface between shallow channel and deep channel-A, figure S3), the pressure difference $\left(\Delta P_{n}\right)$ between inside $\left(P_{a}\right)$ and outside $\left(P_{o}\right)$ the solution is induced and it can be expressed as below: ${ }^{2}$

$$
\Delta P_{n}=P_{a}-P_{0}=-\sigma_{s}\left(\frac{\cos \theta_{T}+\cos \theta_{B}}{h_{s}}+\frac{\cos \left(\theta_{L}+\beta\right)+\cos \left(\theta_{R}+\beta\right)}{w_{t}}\right)
$$

here, $\sigma_{s}, h_{s}$, and $w_{t}$ is the surface tension, the height of shallow channel, and the width of the tip of the shallow channel, respectively. $\Theta$ is the contact angle and the subscripts $T, B, L$, and $R$ indicate the top, bottom, left and right surface of the shallow channel, respectively. $B$ is the expansion angle in the width direction. In these equations, the solution may move forward toward the deep channel-A when the capillary pressure at the neck, $\Delta P_{n}$, is positive, and stop at the neck when $\Delta P_{n}$ is negative.

Here, $\beta$ of $90^{\circ}$ and the value of the surface tension with $0.025 \mathrm{~N} / \mathrm{m}$ was used for $70 \%$ of the ethanol. ${ }^{3}$ In addition, the contact angles were measured from the PDMS substrate (for $\theta_{T}, \theta_{L}$, and $\theta_{R}, \sim 60^{\circ}$ ) and glass substrate (for $\theta_{B}, \sim 45^{\circ}$ ). As a result, the capillary stop pressure at the neck is induced with the values of $-1710 \mathrm{~Pa}$ and the solution could not move forward toward the deep channel-A. 


\section{S1.3 Fabrication process of figure S1(d)}

After the solution stop at the neck, the evaporation of the solvent (70\% of the ethanol) is induced through the deep channel-A. Then the solvent with the convective transport of particles is introduced from the deep channel-B toward the neck to compensate of the solvent loss by evaporation. This influx of the particles promotes the growth of the ordered lattice (FCC structure) from neck to the deep channel-B in unidirectional. However, the careful selection of the size of the nanoparticles and the control of the Debye length, $\lambda_{D}$ (it can be controlled by change of the material or ionic strength, and $\mathrm{pH}$ ) are required because if the nanoparticles have strong repulsive interactions, the assembly to non-FCC structure can happen ${ }^{4}$. When the effective diameter $\left(d_{e f f}\right)$ of the spheres containing Debye length is close to or larger than the center-to-center distance $\left(d_{c}\right)$ between two spheres $\left(d_{\text {eff }}=d+2 \lambda_{D}>d_{c}\right.$, wherein $d$ is the diameter of the colloidal spheres), the colloidal spheres act like "hard spheres" and they will not influence each other until they are in physical contact ${ }^{4,5}$. In this case, the FCC crystal structure is formed, and no heat or energy change is involved upon crystallization.

\section{S1.4 Fabrication process of figure S1(e) and (f)}

(e) When the growth of the micro/nanoparticle assembly reaches the interface between the shallow channel and deep channel-B, the residual solution in deep channel-B is gently removed. Here, the growth of the nanoparticle assembly was easily observed by monitoring the change of the contrast of the light (nanoparticle) in the shallow channel through the inverted microscope (IX7, Olympus Co., Tokyo, Japan). (f) Finally, self-assembled particles are dried out in room temperature for one day. 


\section{S2. Model Calculation}

In this study, we present a numerical model to calculate the ion concentration distribution and ion transport in geometrically asymmetric NCNM by using COMSOL Multiphysics 5.1 with built-in equations. The model was solved by using the "Transport of Diluted Species (Nernst-Planck equation)" and "Electrostatics (Poisson equation)" modules. The variation of the ion concentration distribution and electric potential distribution in nanochannel network membrane under different bulk concentrations and voltage bias was investigated here.

\section{S2.1 Geometry}

In our experiments, we use silica nanoparticles to establish 3-dimentional nanochannel network membrane within the shallow channel as mentioned previously. ${ }^{6}$ The NCNM was designed to be geometrically asymmetrical and fully filled with the selfassembled nanoparticles in a face-centered-cubic (FCC) structure. The nano-interstices formed between these close-packed homogeneously sized nanoparticles, which serve as the pores in the ion-selective membrane, are consist of equivalent nanopores (nanopore size, $d_{n}$, is $\sim 15 \%$ of the sphere size, $\left.D_{n}\right)^{7}$ Therefore, in the case of $240 \mathrm{~nm}$ nanoparticles, the NCNM has pore size of $36 \mathrm{~nm}$, and for $700 \mathrm{~nm}$ nanoparticles, the pore size increases to $105 \mathrm{~nm}$.

In this numerical study, we use a 2D model to simulate the 3D NCNM, because the shallow channel has uniform height from tip-opening to wide-opening. The model illustrated in figure S4 involves NCNM and two reservoirs of electrolyte. The size of shallow channel and deep channel is set to the same dimensions with the actual device. In detail, the widths of the tip-opening $\left(w_{t}\right)$ and wide-opening $\left(w_{w}\right)$ are $10 \mu \mathrm{m}$ and $200 \mu \mathrm{m}$, respectively, and the length of the NCNM $\left(l_{s}\right)$ is $50 \mu \mathrm{m}$ including tip-region $25 \mu \mathrm{m}$ and wide-region $25 \mu \mathrm{m}$. The width and length of the reservoirs are both $200 \mu \mathrm{m}$ and $1000 \mu \mathrm{m}$, respectively. 


\section{S2.2. Governing Equation}

The theoretical model is based on Poisson-Nernst-Planck (PNP) equations. The electric potential is governed by equation $\mathrm{S} 2$ :

$\nabla^{2} \varphi=-\frac{F}{\varepsilon_{0} \varepsilon_{r}} \sum z_{i} c_{i}$

where $\varphi, z$, and $c$ are the electrical potential, the valence and the ion concentration of ion species $i$, respectively. The ion species $i=p / m$, for cations and anions, respectively. $F, \varepsilon_{0}$, and $\varepsilon_{r}$ is the Faraday constant, the permittivity of vacuum, and the dielectric constant of the electrolyte solution as their usual meaning.

The ion transport of potassium and chlorine ions is governed by Nernst-Planck equation:

$j_{i}=D_{i}\left(\nabla c_{i}+\frac{F z_{i} c_{i}}{R T} \nabla \varphi\right)$

and the continuity equation:

$\nabla \cdot j_{i}=0$

where $D$ and $j$ are the diffusion coefficient and ion flux of ion species $i$, respectively. $R$ and $T$ are the gas universal constant and the temperature of solution as their usual meaning. 


\section{S2.3 Parameters and Boundary Conditions}

In this study, different from the single conical nanochannel or nanochannel arrays, the NCNM has nanoporous structure. The diffusivity of ions in porous membrane depends on the molecular dimension, nanopore dimensions, porosity, etc. Therefore, the effective diffusivity of ions within the NCNM will be different from the diffusivity of ions in bulk solution and varies with the pore size of NCNM. As mentioned previously, the nanopore size of NCNM is $36 \mathrm{~nm}$ for $240 \mathrm{~nm}$ nanoparticle, and $105 \mathrm{~nm}$ for $700 \mathrm{~nm}$ nanoparticle. Hence, in this model, the ion diffusion coefficients in the NCNM domain $\left(D_{p m}\right.$ for $\mathrm{K}^{+}$and $D_{m m}$ for $\left.\mathrm{Cl}^{-}\right)$are set to $1 / 5$ and $1 / 2$ of the ion diffusion coefficient in bulk solution, i.e., reservoir $\left(D_{p b}\right.$ for $\mathrm{K}^{+}$and $D_{m b}$ for $\mathrm{Cl}^{-}$) for $36 \mathrm{~nm}$ and $105 \mathrm{~nm}$ nanopore, respectively. ${ }^{8,9}$ The diffusion coefficients for $\mathrm{K}^{+}$and $\mathrm{Cl}^{-}$ ions in this calculation are listed in Table $\mathrm{S} 1$.

The boundary conditions for potential $\varphi$ on the channel wall are given by the Gauss law:

$-\bar{n} \cdot \nabla \varphi=\frac{\sigma}{\varepsilon_{0} \varepsilon_{r}}$

where $\sigma$ is the surface charge density. The negative surface charge density of the silica nanoparticles was reported to be negative $0.01 \mathrm{C} / \mathrm{m}^{2} .{ }^{10}$ In this simulation, the NCNM was regarded as a domain (i.e., volume) not a surface. Therefore, the surface charge density $\sigma$ is calculated into fixed charge concentration $C_{s}$ by using the following equation:

$C_{s}=\frac{4 \pi r^{2} n \sigma}{e N_{a}(1-0.74) V}$ 
here $n=3 / 4 \times 0.74 \times V /\left(\pi r^{3}\right)$ is the total number of nanoparticles in NCNM, and $e, N_{a}, r$ and 0.74 are the charge of electron, the Avogadro's Number, the radii of nanoparticle, and the space occupation rate for close-packed FCC structure which is in dependent of the nanoparticle size. Moreover, as revealed in the experimental data in figure 2, the red intensity was steeply increased from wide to tip and it indicates the distribution of the inhomogeneous fixed charge. Hence, we assumed that this fixed charge distribution, $C(x)$, within NCNM to be given by the following equation (hyperbolic function): ${ }^{11}$

$$
C(x)=\frac{C_{M}}{1-\left(1-C_{M} / C_{m}\right) x / l_{s}}
$$

$C_{M}$ and $C_{m}$ refer to the maximum and minimum values of the fixed charge concentration, respectively, and here the $C_{M}=C_{s}$ and $C_{m}$ was assumed to $0.074 \mathrm{mM}$. In addition, the variation of $x$ is from the tip to the wide opening in NCNM.

The ion flux has the zero normal components at boundaries as shown in figure S4:

$\bar{n} \cdot j=0$

\section{S2.4 Simulation results}

In this simulation, we focused on the nanopore size of $36 \mathrm{~nm}$ because of following reasons: in the visualization experiment, the best conditions for the concentration distribution for diodelike performance were obtained when $w_{t}: w_{w}=10: 200 \mu \mathrm{m}$ with $R \sim 11\left(d_{n}=105 \mathrm{~nm}, \lambda_{D}=\right.$ $\sim 10 \mathrm{~nm}$, and $1 \mathrm{mM} \mathrm{KCl})$. However, to analyze the $I-V$ characteristics, only $\mathrm{K}^{+}$and $\mathrm{Cl}^{-}$ions should be considered, not the fluorescent dyes, so that the size of the nanopore has to be adjusted to match the value of $R$. The size of both $\mathrm{K}+$ and $\mathrm{Cl}-$ ions $(\sim 0.3 \mathrm{~nm})^{12}$ have 
approximately three times smaller than the sizes of the fluorescent dyes (the Alexa-488 and Atto-633 dyes have similar diameters of roughly $\sim 1 \mathrm{~nm}) .{ }^{13,14}$ Therefore, approximately three times smaller nanopores $\left(\sim 36 \mathrm{~nm}, D_{n}=240 \mathrm{~nm}\right)$ and $\lambda_{D}=\sim 3 \mathrm{~nm}(\mathrm{KCl} 10 \mathrm{mM})$ should be considered to match the $R$ close to 11 .

To verify this assume, the simulation was conducted under aforementioned conditions (wherein $R$ is approximately 12 and $w_{t}: w_{w}=10: 200 \mu \mathrm{m}$ ). As a result, the depletion and accumulation of ions were observed in the transition region when the negative and positive potential was applied, respectively (figure S5 (a)). However, when the same condition with $d_{n}=36 \mathrm{~nm}$ case but only the size of nanopore was equal to the visualization study case, i.e. $d_{n}=105 \mathrm{~nm}$, such phenomena became significantly reduced under the same DC bias (figure S5 (b)). In practice, the rectification ratio $\left(f_{\text {rec }}\right)$ at $d_{n}=105 \mathrm{~nm}$ was also significantly reduced $(\sim 1$ at $\pm 10 \mathrm{~V})$ as comparing with $f_{\text {rec }}$ from $d_{n}=36 \mathrm{~nm}$ case $(\sim 55$ at $\pm 10 \mathrm{~V})$.

\section{S3. Reproducibility}

In all experiment, the voltage from a source meter was applied from -10 to $+10 \mathrm{~V}$ in steps of $0.2 \mathrm{~V}$ every $3 \mathrm{~s}$. We repeated the experiment and plotted the $I-V$ curve with error bars. Figure S6 (a), (b), and (c) shows representative $I-V$ plots for the run-to-run results at different samples, especially for $\mathrm{KCl} 10 \mathrm{mM}$, nanoparticle size of $200 \mathrm{~nm}$, and $w_{t}: w_{w}=10: 200 \mu \mathrm{m}$. Each run-to-run experiment was conducted 4 times and used standard deviation. Figure S6 (d) shows the $I-V$ plot for total merged results (i.e. device-to-device result). 


\section{Reference}

1. Chung, S.; Yun, H.; Kamm, R. D. Small 2009, 5, (5), 609-613.

2. Cho, H.; Kim, H.-Y.; Kang, J. Y.; Kim, T. S. J. Colloid Interface Sci. 2007, 306, (2), 379-385.

3. Vazquez, G.; Alvarez, E.; Navaza, J. M. Journal of Chemical \& Engineering Data 1995, 40, (3), 611-614.

4. $\quad$ Xia, Y.; Gates, B.; Yin, Y.; Lu, Y. Adv. Mater. 2000, 12, (10), 693-713.

5. $\quad$ Okubo, T. Langmuir 1994, 10, (6), 1695-1702.

6. $\quad$ Choi, E.; Kwon, K.; Kim, D.; Park, J. Lab on a chip 2015, 15, (1), 168-78.

7. Zeng, Y.; Harrison, D. J. Anal Chem 2007, 79, (6), 2289-2295.

8. Shen, M.; Yang, H.; Sivagnanam, V.; Gijs, M. Anal Chem 2010, 82, (24), 9989-9997.

9. Dhopeshwarkar, R.; Crooks, R. M.; Hlushkou, D.; Tallarek, U. Anal Chem 2008, 80, (4), 1039-1048.

10. Choi, E.; Kwon, K.; Kim, D.; Park, J. Lab on a chip 2015, 15, (2), 512-23.

11. Ramirez, P.; Gomez, V.; Cervera, J.; Schiedt, B.; Mafe, S. J Chem Phys 2007, 126, (19), 194703.

12. Volkov, A. G.; Paula, S.; Deamer, D. W. Bioelectrochemistry and Bioenergetics 1997, 42, (2), 153-160.

13. Masuda, A.; Ushida, K.; Okamoto, T. Biophysical Journal 2005, 88, (5), 3584-3591.

14. Illaste, A.; Laasmaa, M.; Peterson, P.; Vendelin, M. Biophysical Journal 2012, 102, (4), 739-48. 


\section{Figures and legends}

(a)

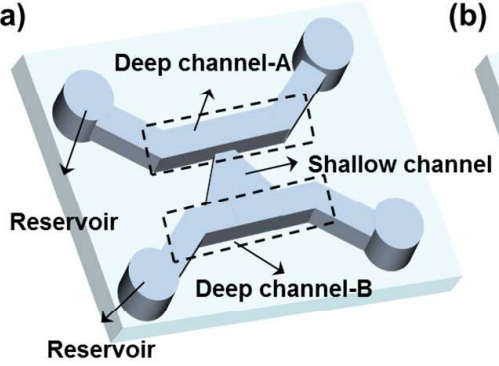

(b)

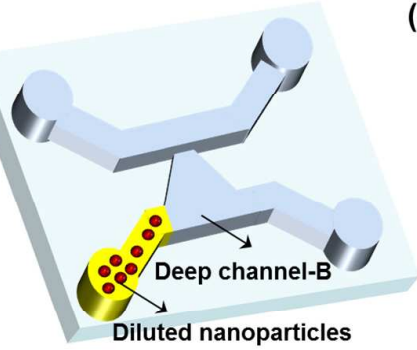

(c)

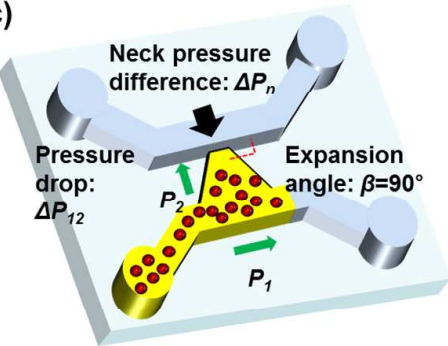

(d)

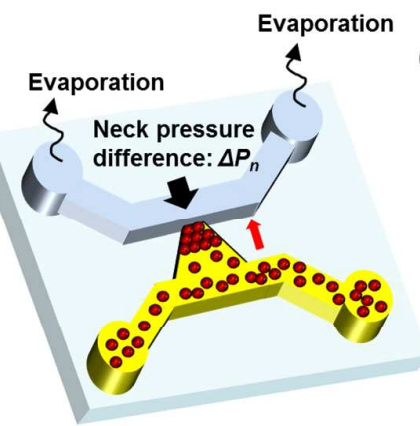

(e)

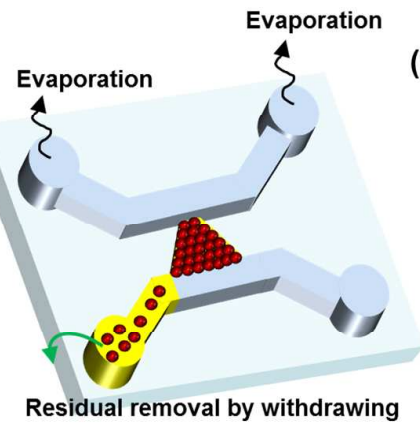

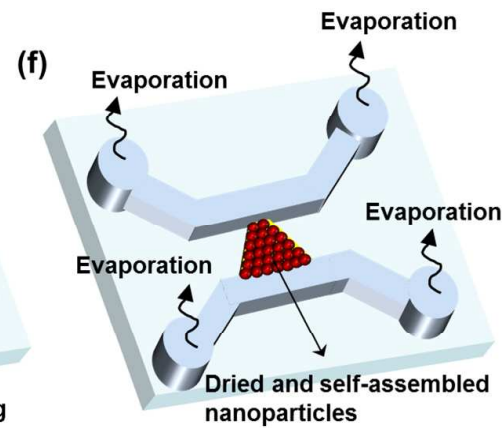

Figure S1. Fabrication process for the in situ formation of nanochannel networks membranes using the self-assembly of particles within the PDMS channel

(a)

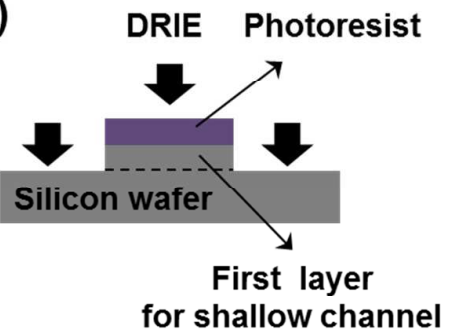

(b)

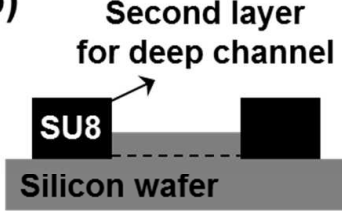

(c)

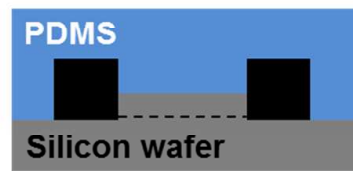

(d)

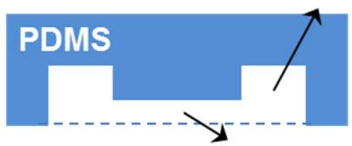

Shallow channel (e)

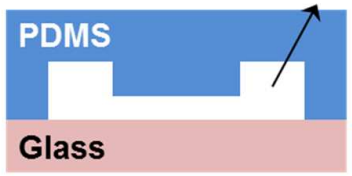

Figure S2. Scheme of the fabrication process for PDMS microfluidic channels with multilayered design. 
$<$ Top view>

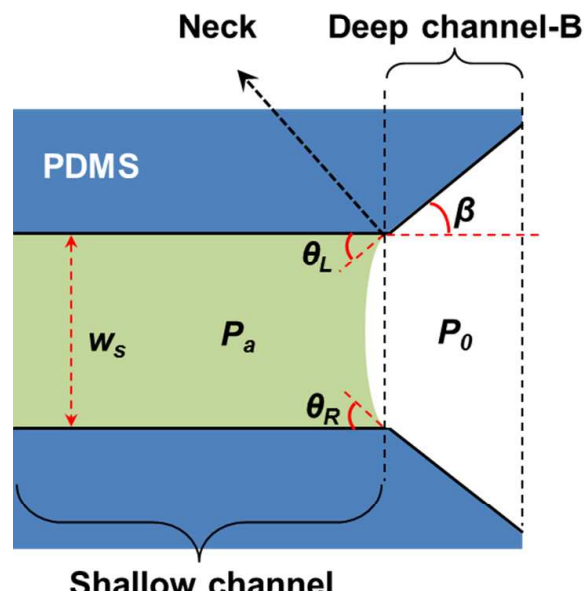

<Side view>

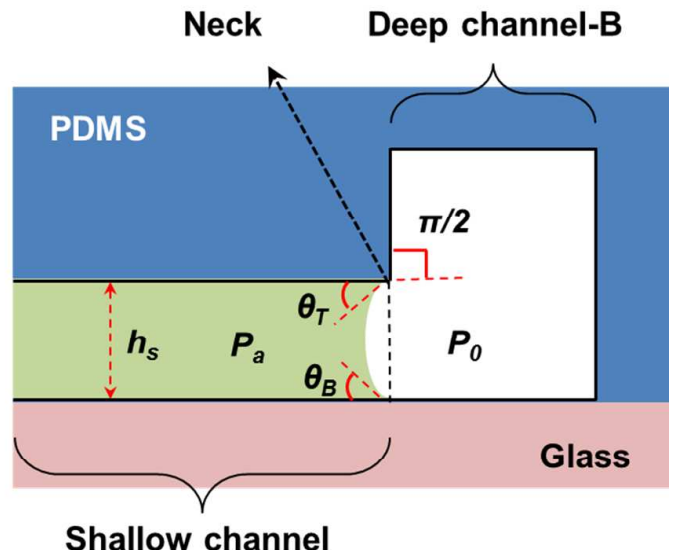

Figure S3. Schematic of the capillary flow of diluted nanoparticles at the neck of the shallow channel. 


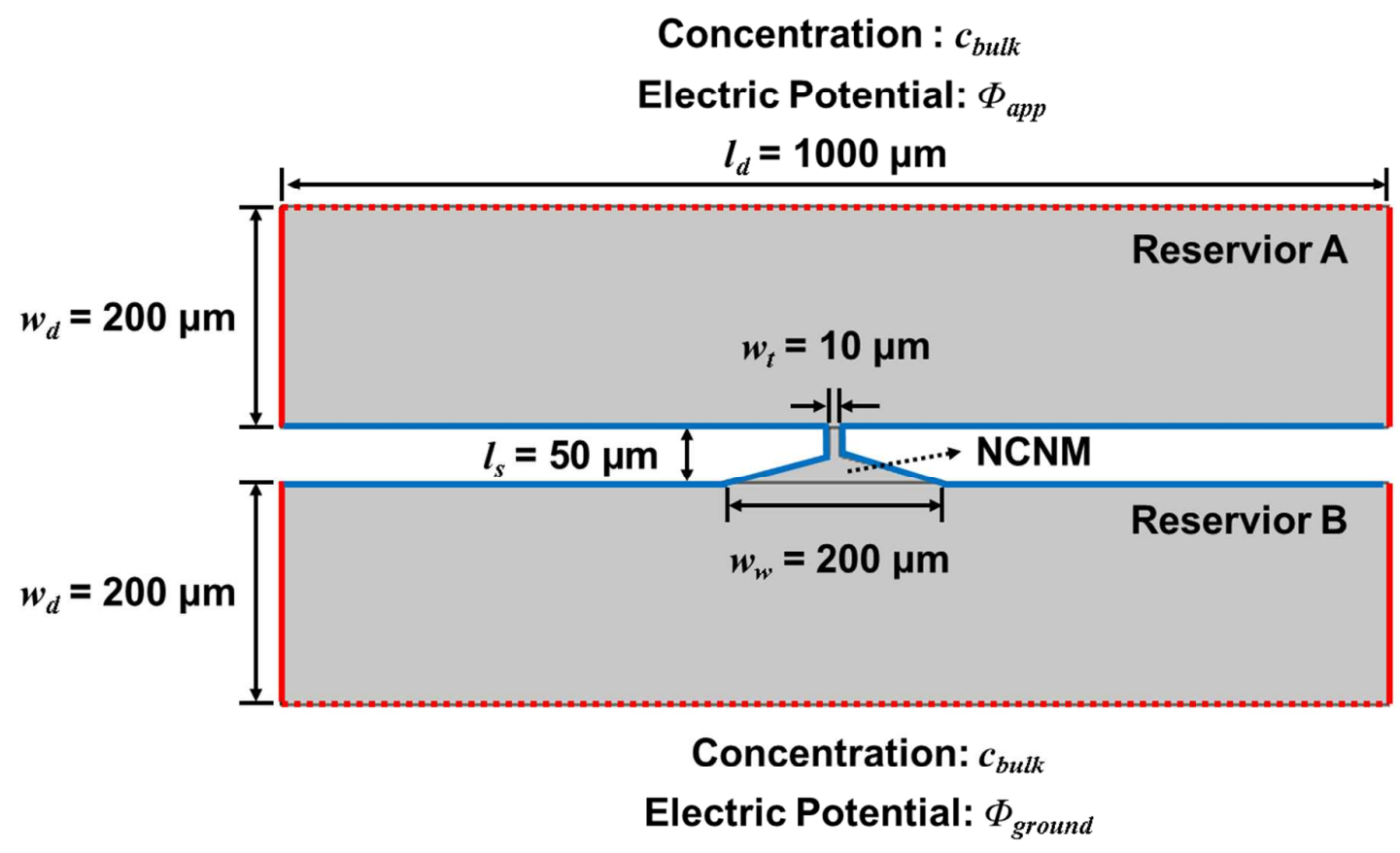

Figure S4. The geometry of the model. $w_{d}, l_{d}, w_{t}, w_{w}$, and $l_{s}$ are the width of the deep channel (reservoir), the length of the deep channel (reservoir), the width of the tip-opening, the width of the wide-opening, and the length of the shallow channel (NCNM), respectively. $c_{b u l k}, \Phi_{a p p}$, and $\Phi_{\text {ground }}$ are the bulk concentration, the voltage bias at the reservoir A, and the voltage bias at the reservoir B, respectively. Concentration $c_{b u l k}$ is set to the channel walls of reservoirs marked with red dashed lines, and the electric potential $\Phi_{\text {app }}$ and $\Phi_{\text {ground }}$ are applied to the channel walls of reservoir A and B, respectively, marked with red solid lines. The zero normal flux are set to the channel walls marked with blue solid lines. In the simulation, the electric potential $\Phi_{\text {ground }}$ applied at the channel wall of the reservoir B is set to be zero. And the electric potential $\Phi_{a p p}$ applied at the channel wall of the reservoir A is calculated with electric field $E=U / d$, where $U$ and $d$ are the potential difference and the distance between electrodes, respectively. The values of $U$ and $d$ are $10 \mathrm{~V}$ and $8.5 \mathrm{~mm}$ in the experiment, and $1.2 \mathrm{~V}$ and $1.05 \mathrm{~mm}$ in the simulation, respectively. 
(a) $\mathrm{KCl} 10 \mathrm{mM}$, Nanopore size: $36 \mathrm{~nm}$
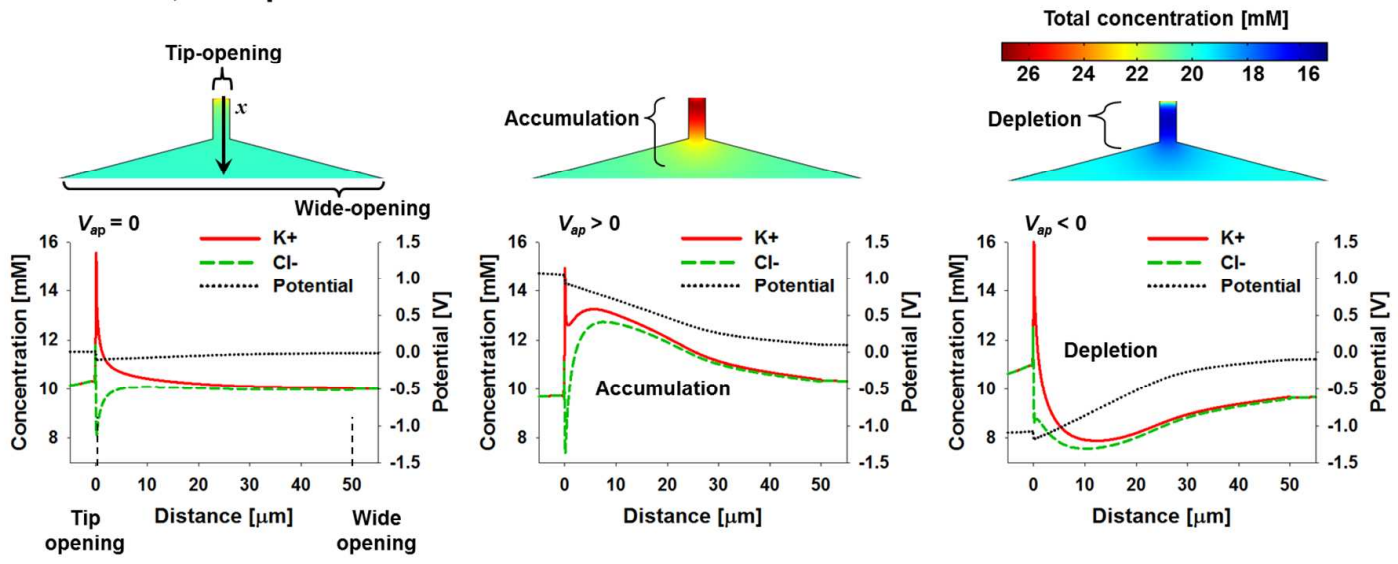

(b) $\mathrm{KCl} 10 \mathrm{mM}$, Nanopore size: $105 \mathrm{~nm}$
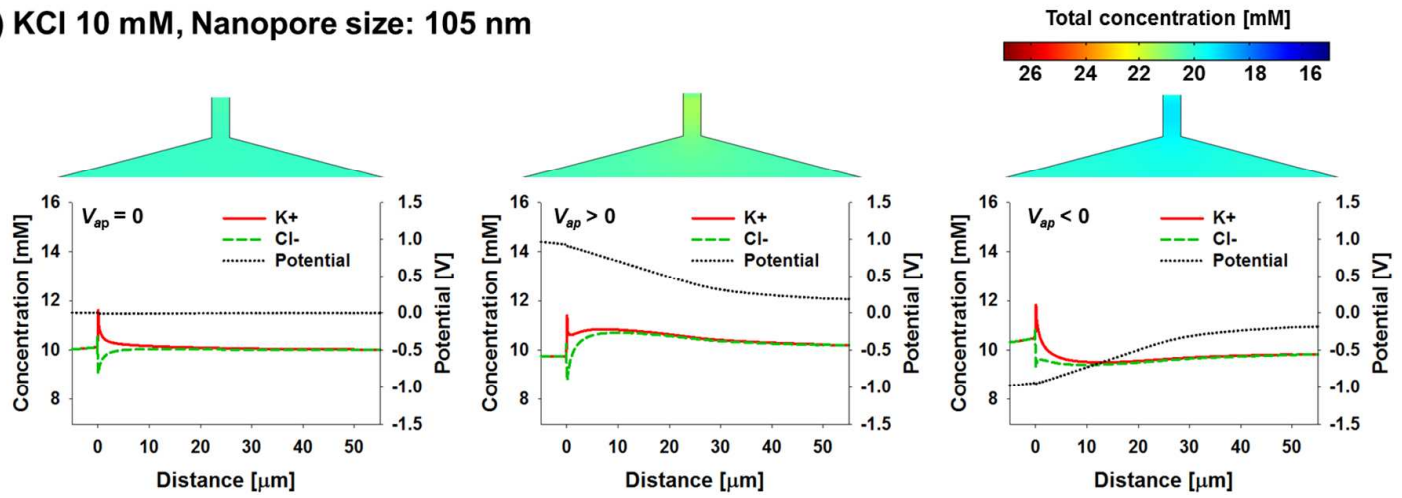

Figure S5. Total concentration distribution images in NCNM and related ions concentration/potential distribution plot from the numerical simulation. The condition was (a) $\mathrm{KCl} 10 \mathrm{mM}$ and nanopore size of $36 \mathrm{~nm}$ and (b) $\mathrm{KCl} 10 \mathrm{mM}$ and nanopore size of $105 \mathrm{~nm}$. 
(a)

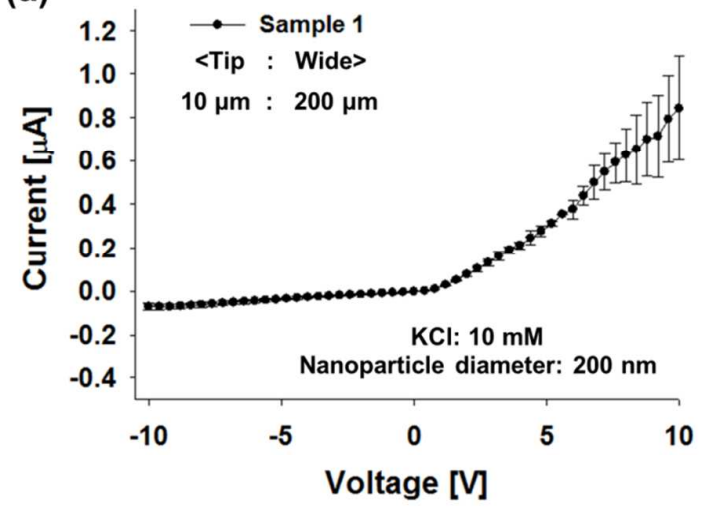

(c)

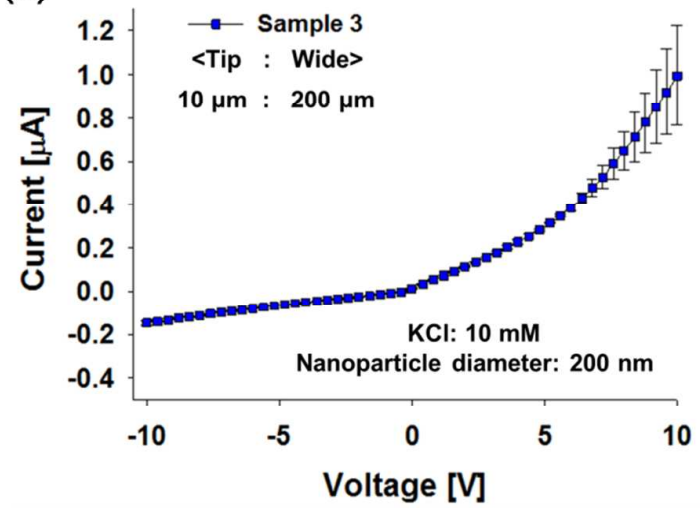

(b)

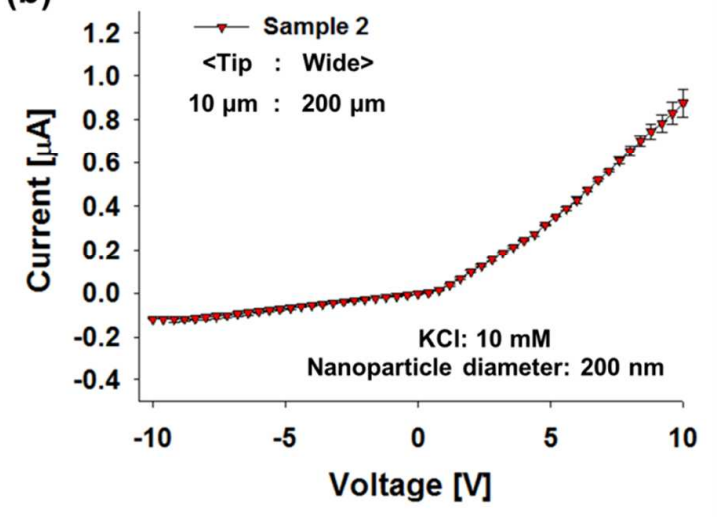

(d)

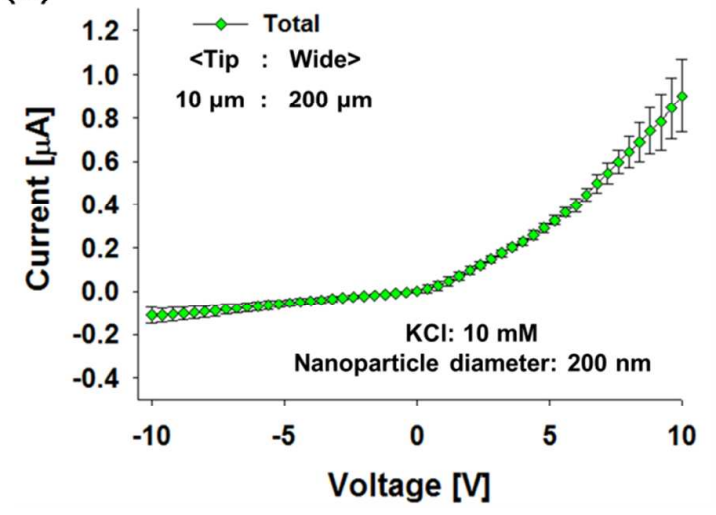

Figure S6. (a), (b), and (c) representative $I-V$ plots for the run-to-run results at different samples, especially for $\mathrm{KCl} 10 \mathrm{mM}$, nanoparticle size of $200 \mathrm{~nm}$, and $w_{t}: w_{w}=10: 200 \mu \mathrm{m}$. Each run-to-run experiment was conducted 4 times and used standard deviation. (d) $I-V$ plot for total merged results (i.e. device-to-device result). 
Table S1. Diffusivities of ions in NCNM and reservoirs used in the model.

\begin{tabular}{cccc}
\hline \multirow{2}{*}{ Nanopore Size } & Ion Species $i$ & $\begin{array}{l}\text { Diffusivity in } \\
\text { Reservoir, } D_{i b}\end{array}$ & $\begin{array}{c}\text { Diffusivity in } \\
\text { NCNM, } D_{i m}\end{array}$ \\
\hline \multirow{2}{*}{$36 \mathrm{~nm}$} & $\mathrm{~K}^{+}$ & $1.957 \mathrm{e}-9 \mathrm{~m}^{2} / \mathrm{s}$ & $3.914 \mathrm{e}-10 \mathrm{~m}^{2} / \mathrm{s}$ \\
\cline { 2 - 4 } & $\mathrm{Cl}^{-}$ & $2.032 \mathrm{e}-9 \mathrm{~m}^{2} / \mathrm{s}$ & $4.064 \mathrm{e}-10 \mathrm{~m}^{2} / \mathrm{s}$ \\
\hline \multirow{2}{*}{$105 \mathrm{~nm}$} & $\mathrm{~K}^{+}$ & $1.957 \mathrm{e}-9 \mathrm{~m}^{2} / \mathrm{s}$ & $9.785 \mathrm{e}-10 \mathrm{~m}^{2} / \mathrm{s}$ \\
\cline { 2 - 4 } & $\mathrm{Cl}^{-}$ & $2.032 \mathrm{e}-9 \mathrm{~m}^{2} / \mathrm{s}$ & $1.016 \mathrm{e}-9 \mathrm{~m}^{2} / \mathrm{s}$ \\
\hline
\end{tabular}

